\title{
ON THE \\ LOCAL REGULARIZATION OF INVERSE PROBLEMS OF VOLTERRA TYPE
}

\author{
Patricia K. Lamm \\ Department of Mathematics \\ Michigan State University \\ East Lansing, Michigan
}

\begin{abstract}
We consider a local regularization method for the solution of first-kind Volterra integral equations with convolution kernel. The local regularization is based on a splitting of the original Volterra operator into "local" and "global" parts, and a use of Tikhonov regularization to stabilize the inversion of the local operator only. The regularization parameters for the local procedure include the standard Tikhonov parameter, as well as a parameter that represents the length of the local regularization interval. We present a convergence theory for the infinite-dimensional regularization problem and show that the regularized solutions converge to the true solution as the regularization parameters go to zero (in a prescribed way). In addition, we show how numerical implementation of the ideas of local regularization can lead to the notion of "sequential Tikhonov regularization" for Volterra problems; this approach has been shown in (Lamm and Eldén, 1995) to be just as effective as Tikhonov regularization, but to be much more efficient computationally.
\end{abstract}

\section{INTRODUCTION}

We consider a local regularization method for the problem of finding $u$ solving

$$
A u=f
$$

where $A u(t)=\int_{0}^{t} k(t-\tau) u(\tau) d \tau, 0 \leq t \leq 1$, is a first-kind Volterra integral operator with convolution kernel $k$ satisfying $k(t)>0$ on $(0,1], k$ uniformly Lipschitz continuous on $[0,1]$ with Lipschitz constant $L_{k}$. We assume throughout that the "ideal" data $f$ is also uniformly Lipschitz continuous on $[0,1]$ (with Lipschitz constant $L_{f}$ ), and assume $f$ is sufficiently smooth to guarantee a unique solution $u \in L_{2}(0,1)$ of (1) (see, e.g., (Gripenberg, 1990)).

Of particular interest is the typical case which occurs in applications, namely, the situation where we only have available perturbed data $f^{\delta} \in L_{2}(0,1)$; here $f^{\delta}(t)=f(t)+d(t)$ where $\|d\|<\delta$ for some $\delta>0$ and $\|\cdot\|$ is the $L_{2}$ norm. Because solutions of (1) are unstable with respect to error in the data, a regularizing method is needed to solve the perturbed problem.

\section{LOCAL REGULARIZATION}

We present here a local regularization method that is ideally suited for Volterra problems of convolution type, but also has extensions (to be discussed elsewhere) to non-Volterra, non-convolution problems.

In order to simplify our presentation in what follows we assume that equation (1) holds on an extended time interval $[0,1+\Delta R]$, where $\Delta R>0$ is fixed and small, and that the perturbed data $f^{\delta}$ is available on this extended interval as well; a straightforward modification made be made in the theory to handle the case where this assumption is not made (e.g., see (Lamm, 1995)).

The idea of local regularization we consider is based 
on a splitting of the operator $A$ into "local" and "global" parts, while performing standard Tikhonov regularization directly on the local part only. To consider this approach in the context of the Volterra problem, we first note that for fixed $\Delta r \in[0, \Delta R]$ and a.a. $t \in[0,1]$, $\rho \in[0, \Delta r]$,

$$
\begin{aligned}
& f(t+\rho)=A u(t+\rho) \\
& \quad=\int_{0}^{\rho} k(\rho-s) u(t+s) d s+\int_{0}^{t} k(t+\rho-s) u(s) d s \\
& \quad=\mathcal{A}_{\Delta r} u_{\Delta r}(t)(\rho)+\mathcal{B}_{\Delta r} u(t)(\rho),
\end{aligned}
$$

where

$$
u_{\Delta r}(t)(\rho)=u(t+\rho)
$$

for a.a $t \in[0,1], \rho \in[0, \Delta r]$, and where $\mathcal{A}_{\Delta r}$ and $\mathcal{B}_{\Delta r}$ will be defined more precisely below. In general, for a given value of $t$, the quantity $\mathcal{A}_{\Delta r} u_{\Delta r}(t)$ represents the action of the original operator $A$ on the solution $u$ in the local interval $[t, t+\Delta r]$, while $\mathcal{B}_{\Delta r} u(t)$ represents the action of $A$ on $u$ over the more global (and "past") interval $[0, t]$. (We note that the "local interval" associated with the value $t$ used here is $[t, t+\Delta r]$, rather than $[t-\Delta r, t+\Delta r]$, because we are considering a Volterra, i.e., causal, problem.)

Next we define $\Delta r$-dependent spaces and give precise definitions of the $\Delta r$-dependent operators used above. For $\Delta r \in(0, \Delta R]$, we denote by $X_{\Delta r}=L_{2}((0, \Delta r) ; 1 / \Delta r)$ the usual $L_{2}(0, \Delta r)$ space with weighted inner product, $(\psi, \chi)_{\Delta r}=\frac{1}{\Delta r} \int_{0}^{\Delta r} \psi(s) \chi(s) d s$, and define the following $\Delta r$-dependent spaces,

$$
\begin{aligned}
& Z_{\Delta_{r}}=L_{2}\left((0,1), X_{\Delta_{r}}\right) \\
& H_{\Delta_{r}}=L_{2}(0,1) \times Z_{\Delta r},
\end{aligned}
$$

where $L_{2}\left((0,1), X_{\Delta r}\right)$ denotes the square-integrable $X_{\Delta r}$-valued "functions" on $[0,1]$. We also define fixed spaces $X, Z$, and $H$ by considering the above definitions in the case of $\Delta r=1$ and using the notation $X \equiv X_{1}, Z \equiv Z_{1}$, and $H \equiv H_{1}$.

The operators $\mathcal{A}_{\Delta r}$ and $\mathcal{B}_{\Delta r}$ are given as follows:

$$
\begin{aligned}
& \mathcal{A}_{\Delta r} \in \mathcal{L}\left(Z_{\Delta r}, Z_{\Delta r}\right),=\int_{0}^{\rho} k(\rho-s) \varphi(t)(s) d s \\
& \mathcal{A}_{\Delta r} \varphi(t)(\rho)=\int_{0} k\left(L_{2}(0,1), Z_{\Delta r}\right), \\
& \mathcal{B}_{\Delta r} \in \mathcal{L}(t+\rho-s) \eta(s) d s,
\end{aligned}
$$

for $\eta \in L_{2}(0,1), \varphi \in Z_{\Delta r}$, and where $\mathcal{L}(X, Y)$ denotes the space of bounded linear operators from $X$ to $Y$. We note that $\mathcal{A}_{\Delta r}$ and $\mathcal{B}_{\Delta r}$ have operator norms $\left\|\mathcal{A}_{\Delta r}\right\|=$ $\mathcal{O}(\Delta r)$ and $\left\|\mathcal{B}_{\Delta r}\right\|=\mathcal{O}(1)$, respectively, as $\Delta r \rightarrow 0$. In addition, for each $\Delta r \in(0, \Delta R]$, we define the operator $\mathcal{C}_{\Delta r}$ by

$$
\begin{gathered}
\mathcal{C}_{\Delta r} \in \mathcal{L}\left(H_{\Delta r}, Z_{\Delta r}\right) \\
\mathcal{C}_{\Delta r}(\eta, \varphi)=\mathcal{B}_{\Delta r} \eta+\mathcal{A}_{\Delta r} \varphi, \quad(\eta, \varphi) \in H_{\Delta r}
\end{gathered}
$$

and define $F_{\Delta r} \in Z_{\Delta r}$ by

$$
F_{\Delta r}(t)(\rho)=f(t+\rho), \quad 0 \leq t \leq 1,0 \leq \rho \leq \Delta r
$$

making a similar definition for $F_{\Delta r}^{\delta} \in Z_{\Delta r}$ using the perturbed data $f^{\delta}$.

The "local" Tikhonov regularization problem that we consider is given as follows:

Problem $\mathcal{P}_{\Delta r}$ :

$$
\begin{array}{r}
\min \left\{\left\|\mathcal{C}_{\Delta r}(\eta, \varphi)-F_{\Delta r}^{\delta}\right\|_{\Delta r}^{2}+\alpha\left\|\mathcal{L}_{\Delta r} \varphi\right\|_{\Delta r}^{2}:\right. \\
\left.(\eta, \varphi) \in D_{\Delta_{r}}, \varphi \in \operatorname{dom}\left(\mathcal{L}_{\Delta r}\right)\right\}
\end{array}
$$

where $\|\cdot\|_{\Delta_{r}}$ denotes the (weighted) norm on $Z_{\Delta_{r}}$. Here $\mathcal{L}_{\Delta r}: \operatorname{dom}\left(\mathcal{L}_{\Delta r}\right) \subset Z_{\Delta r} \mapsto Z_{\Delta r}$ denotes a suitable closed operator, and $D_{\Delta r} \subset H_{\Delta r}$ is a constraint set which links $\eta(t)$ to $\varphi(t)(\cdot)$ in an essential way. The "localized regularization" comes about in the direct regularization of $\varphi$ via $\mathcal{L}_{\Delta r}$; the variable $\eta$ is regularized indirectly via the constraint $(\eta, \varphi) \in D_{\Delta r}$ (and in fact $\eta$ is the regularized approximation to $u$ that we seek).

In particular, let $L_{\Delta r}: \operatorname{dom}\left(L_{\Delta r}\right) \subset X_{\Delta_{r}} \rightarrow X_{\Delta r}$ be a closed operator, and define $\mathcal{L}_{\Delta r}$ as follows:

$$
\mathcal{L}_{\Delta r} \varphi(t)(\rho)=L_{\Delta r}(\varphi(t))(\rho),
$$

where $\varphi \in \operatorname{dom}\left(\mathcal{L}_{\Delta r}\right), 0 \leq t \leq 1,0 \leq \rho \leq \Delta r$, and where

$$
\begin{gathered}
\operatorname{dom}\left(\mathcal{L}_{\Delta_{r}}\right)=\left\{\varphi \in Z_{\Delta r}:\right. \\
\varphi(t) \in \operatorname{dom}\left(L_{\Delta r}\right) \text { for a.a. } t \in[0,1], \\
\left.\int_{0}^{1}\left\|L_{\Delta_{r}}(\varphi(t))\right\|_{X_{\Delta r}}^{2} d t<\infty\right\} .
\end{gathered}
$$

As for the definition of $D_{\Delta r}$, ideally $(\eta, \varphi) \in D_{\Delta r}$ should imply that $\eta(t)=\varphi(t)(0)$ for a.a. $t \in[0,1]$ (i.e., the condition satisfied by $\left.\left(u, u_{\Delta r}\right)\right)$. But point evaluations require $\varphi(t) \in C[0,1]$, regularity which does not occur in general for $\varphi \in \operatorname{dom}\left(\mathcal{L}_{\Delta r}\right)$. In order to handle the general case, we take an alternate approach here in the formulation of $D_{\Delta r}$.

To this end we let $\ell$ denote a bounded linear functional on $X$ which satisfies the condition that $\ell(\chi)=1$ 
when $\chi \in X$ implies $\chi(x) \equiv 1, x \in[0,1]$. Let $\gamma \in X$ be uniquely given by $\ell(\psi)=(\psi, \gamma)$ for all $\psi \in X$, where $(\cdot, \cdot)$ denotes the $X=L_{2}(0,1)$ inner product; we will assume throughout that $\gamma \in L_{\infty}(0,1)$ (a condition which can be dropped if the true solution $u$ of $(1)$ is in $\left.L_{\infty}(0,1)\right)$. We then define the bounded linear operator $T: Z \rightarrow L_{2}(0,1)$ by

$$
T \varphi(t)=\ell(\varphi(t)), \quad t \in[0,1]
$$

for $\varphi \in Z$. In addition, $\Delta r$-dependent versions of $\ell$ and $T$ are defined for each $\Delta r \in(0, \Delta R]$ by

$$
\begin{aligned}
\ell_{\Delta r} & \in \mathcal{L}\left(X_{\Delta r}, \mathbb{R}\right), \\
\ell_{\Delta r}(\psi) & =\ell(\tilde{\psi}), \quad \psi \in X_{\Delta r}
\end{aligned}
$$

where $\tilde{\psi} \in X$ is defined from $\psi \in X_{\Delta r}$ via $\tilde{\psi}(\rho)=$ $\psi(\rho \Delta r), 0 \leq \rho \leq 1$; in addition, we define

$$
\begin{aligned}
T_{\Delta r} & \in \mathcal{L}\left(Z_{\Delta r}, L_{2}(0,1)\right), \\
T_{\Delta r} \varphi(t) & =\ell_{\Delta r}(\varphi(t)), \quad \varphi \in Z_{\Delta r} .
\end{aligned}
$$

We note that the operators defined above satisfy, in their respective operator topologies, $\left\|T_{\Delta r}\right\|=\left\|\ell_{\Delta r}\right\|=$ $\|\ell\|=\|\gamma\|_{X}$.

One example motivating our definition of $\ell$ is

$$
\ell \psi=\frac{1}{c} \int_{0}^{c} \psi(\rho) d \rho, \quad \psi \in X,
$$

where $c \in(0,1]$. In this case we note that $T_{\Delta r}$ is given in particular on $u_{\Delta r}$ for each $t \in[0,1]$ by

$$
T_{\Delta r} u_{\Delta r}(t)=\frac{1}{c \Delta r} \int_{t}^{t+c \Delta r} u(s) d s
$$

i.e., the average value of $u$ over the (small) interval $[t, t+c \Delta r]$. (We also note that in the definitions given above, both $\ell$ and $T$ could have been $\Delta r$-dependent, a consideration which is not taken here.)

Using $\ell_{\Delta r}$ and $T_{\Delta r}$, we define

$$
D_{\Delta r}=\left\{(\eta, \varphi) \in H_{\Delta r}: \eta=T_{\Delta r} \varphi\right\}
$$

a closed subspace of $H_{\Delta r}$. Then standard assumptions on the operators in problem $\mathcal{P}_{\Delta r}$ (see Theorem 1 below) guarantee that there is a unique solution $\left(\eta_{\alpha, \Delta r}^{\delta}, \varphi_{\alpha, \Delta r}^{\delta}\right)$ of problem $\mathcal{P}_{\Delta r}$ for each $\alpha>0, \delta>0$ and $\Delta r \in(0, \Delta R]$. Our goal is to obtain conditions on the pair $\{\alpha, \Delta r\}$ such that $\eta_{\alpha, \Delta r}^{\delta} \rightarrow u$ in $L_{2}(0,1)$ as each of $\Delta r, \alpha, \delta$ converges to zero in a prescribed way.
As an initial study into the problem of local regularization, in this paper we restrict our consideration in this paper to the case of $L_{\Delta r}=I_{\Delta r}$, the identity operator on $X_{\Delta_{r}}$; i.e., $\mathcal{L}_{\Delta_{r}} \varphi(t)(\rho)=\varphi(t)(\rho)$ for $\varphi \in \operatorname{dom}\left(\mathcal{L}_{\Delta r}\right) \equiv Z_{\Delta r}$. We first describe the numerical algorithm that arises in this case, indicating how a rapid "sequential Tikhonov regularization" method naturally occurs as a result.

\section{NUMERICAL IMPLEMENTATION}

We discuss here the implementation of the above regularization procedure for Volterra problems and indicate the way in which the "local" nature of the procedure is exhibited. The numerical discretization will be described here in terms of a collocation scheme for the integral equation (with solutions approximated by piecewise constants), but it is expected that similar comments also hold for methods based on numerical quadrature of the integrals involved (as is true, for example, in (Lamm and Eldén, 1995)).

Let integers $M \geq 0$ and $R \geq 1$ be fixed, and define $\Delta t=1 /(M+1), \Delta r=R \Delta t$, and $t_{i}=i \Delta t$, $i=0,1, \ldots, M+R+1$. In order to discretize the minimization problem $\mathcal{P}_{\Delta r}$, we search for $(\eta, \varphi) \in H_{\Delta r}$ of the special form

$$
\begin{aligned}
& \varphi(t)(\rho)= \sum_{i=0}^{M} \sum_{j=0}^{R} c_{i j} \chi_{i}(t) \chi_{j}(\rho), \\
& t \in[0,1], \rho \in[0, \Delta r], \\
& \eta(t)= c_{i 0} \chi_{i}(t), \quad t \in\left[t_{i}, t_{i+1}\right),
\end{aligned}
$$

where $c_{i j}, i=0, \ldots, M, j=0, \ldots, R$, are unknown constants to be determined, and where

$$
\chi_{i}(t)= \begin{cases}1, & t \in\left[t_{i}, t_{i+1}\right) \\ 0, & \text { otherwise }\end{cases}
$$

We note that $\eta(t)=\ell_{\Delta r}(\varphi(t))$ where $\ell_{\Delta r}$ is defined as usual from $\ell$, and where $\ell$ in this case is given by (4) using $c=1 / R<1$.

In this finite-dimensional setting, we seek the matrix c of unknowns $\left(\mathbf{c}=\left(c_{i j}\right)_{i=0, \ldots M, j=0, \ldots R}\right)$ which minimizes the functional $J_{M, R}(\mathbf{c})$,

$$
\begin{aligned}
& J_{M, R}(\mathbf{c})= \\
& \sum_{m=0}^{M} \sum_{r=0}^{R} \mid \int_{0}^{\rho_{r+1}} k\left(\rho_{r+1}-s\right) \sum_{i=0}^{M} \sum_{j=0}^{R} c_{i j} \chi_{i}\left(t_{m}\right) \chi_{j}(s) d s \\
& \quad+\int_{0}^{t_{m}} k\left(t_{m}+\rho_{r+1}-s\right) \sum_{i=0}^{M} c_{i 0} \chi_{i}(s) d s-\left.f\left(t_{m}+\rho_{r+1}\right)\right|^{2}
\end{aligned}
$$




$$
+\alpha \sum_{i=0}^{M} \sum_{j=0}^{R}\left|c_{i j}\right|^{2}
$$

which corresponds to a collocation-based discretization (collocating to points $\left(t_{m}, \rho_{r+1}\right), m=0,1, \ldots, M$, $\left.\rho_{r+1} \equiv t_{r+1}, r=0,1, \ldots, R\right)$ of the objective functional appearing in problem $\mathcal{P}_{\Delta r}$. Due to the Volterra (convolution) form of the problem, $J_{M, R}$ simplifies considerably, i.e.,

$$
\begin{aligned}
J_{M, R}(\mathbf{c})= & \sum_{m=0}^{M} \sum_{r=0}^{R} \mid \sum_{j=0}^{r} c_{m j} \Delta_{r+1-j} \\
& +\sum_{i=0}^{m-1} c_{i 0} \Delta_{m+r+1-i}-\left.f\left(t_{m+r+1}\right)\right|^{2} \\
& +\alpha \sum_{i=0}^{M} \sum_{j=0}^{R}\left|c_{i j}\right|^{2},
\end{aligned}
$$

where $\Delta_{i} \equiv \int_{0}^{\Delta t} k\left(t_{i}-s\right) d s$. If we make the following definitions for $m=0,1, \ldots, M$,

$$
\begin{aligned}
\mathbf{c}_{m} & \equiv\left(c_{m 0}, c_{m 1}, \ldots, c_{m R}\right)^{\top} \in \mathbb{R}^{R+1}, \\
\mathbf{f}_{m} & \equiv\left(f\left(t_{m+1}\right), \ldots, f\left(t_{m+R+1}\right)\right) \in \mathbb{R}^{R+1},
\end{aligned}
$$

as well as the definition of the $(R+1)$-square matrix $K_{R}$,

$$
K_{R}=\left(\begin{array}{cccc}
\Delta_{1} & 0 & \ldots & 0 \\
\Delta_{2} & \Delta_{1} & \ldots & 0 \\
\vdots & \vdots & \ddots & \vdots \\
\Delta_{R+1} & \Delta_{R} & \ldots & \Delta_{1}
\end{array}\right)
$$

the finite-dimensional optimization problem becomes

$$
\min _{\mathbf{c}_{0}, \ldots, \mathbf{c}_{M}} \sum_{m=0}^{M} J_{m, R}\left(\mathbf{c}_{0}, \ldots, \mathbf{c}_{m}\right)
$$

where

$$
\begin{aligned}
& J_{m, R}\left(\mathbf{c}_{0}, \ldots, \mathbf{c}_{m}\right)= \\
& \left\|K_{R} \mathbf{c}_{m}+\sum_{i=0}^{m-1} c_{i 0} \hat{\Delta}_{m-i+1}-\mathbf{f}_{m}\right\|_{R+1}^{2}+\alpha\left\|\mathbf{c}_{m}\right\|_{R+1}^{2},
\end{aligned}
$$

where here $\|\cdot\|_{R+1}$ denotes the usual $\mathbb{R}^{R+1}$ norm and $\hat{\Delta}_{i} \equiv\left(\Delta_{i}, \ldots, \Delta_{i+R}\right)^{\top}$ for given $i$.

Using the theory of (Auslender, 1971), a decomposition of this minimization problem is possible, setting up an iterative relaxation-type minimization. The following algorithm finds $\mathbf{c}_{I}$ for $I=0,1, \ldots, M$, using at each step $\beta \in \mathbb{R}^{R+1}$ to store the unknown $\mathbf{c}_{I}$. The vectors $\mathbf{d}_{0}, \mathbf{d}_{1}, \ldots, \mathbf{d}_{M}$ in $\mathbb{R}^{R+1}$ used below may be initialized to zero and correspond to initial guesses for $\mathbf{c}_{0}, \mathbf{c}_{1}, \ldots, \mathbf{c}_{M}$.

\section{Local Tikhonov Regularization Algorithm \#1}

1. Initialize vectors $\mathbf{d}_{0}, \mathbf{d}_{1}, \ldots, \mathbf{d}_{M}$.

2. Let $I=0$.

3. Holding the previously determined values of $\mathbf{c}_{0}$, $\mathbf{c}_{1}, \ldots, \mathbf{c}_{I-1}$ fixed, find $\bar{\beta}$ solving

$$
\begin{aligned}
\min _{\beta} & \left\{\sum_{m=0}^{I-1} J_{m, R}\left(\mathbf{c}_{0}, \ldots, \mathbf{c}_{m}\right)\right. \\
& +J_{I, R}\left(\mathbf{c}_{0}, \ldots, \mathbf{c}_{I-1}, \beta\right) \\
& \left.+\sum_{m=I+1}^{M} J_{m, R}\left(\mathbf{c}_{0}, \ldots, \mathbf{c}_{I-1}, \beta, \mathbf{d}_{I+1}, \ldots \mathbf{d}_{m}\right)\right\}
\end{aligned}
$$

(i.e., $\mathbf{c}_{I+1}=\mathbf{d}_{I+1}, \ldots, \mathbf{c}_{m}=\mathbf{d}_{m}$ temporarily).

4. Set $\mathbf{c}_{I}=\bar{\beta}$.

5. If $I=M$, let $\mathbf{d}_{i}=\mathbf{c}_{i}$ for $i=0, \ldots, M$, and return to step 3. Otherwise, leave the $\mathbf{d}_{i}$ unchanged, increment $I$ by 1 and return to step 3 .

Convergence is guaranteed (Auslender, 1971), and the converged values $c_{00}, c_{10}, \ldots, c_{M 0}$ are approximations for $u\left(t_{0}\right), u\left(t_{1}\right), \ldots, u\left(t_{M}\right)$.

Remark: For $R=R(\delta)$ appropriately chosen (given the level $\delta$ of noise in the problem), it has been observed in practice that very accurate results are obtained if, instead of solving (7) for $\bar{\beta}$ in step 3 above, one instead finds $\bar{\beta}$ solving

$$
\min _{\beta} J_{I, R}\left(\mathbf{c}_{0}, \ldots, \mathbf{c}_{I-1}, \beta\right) .
$$

Since $J_{I, R}$ is independent of $\mathbf{d}_{I+1}, \ldots, \mathbf{d}_{M}$, the entire iteration for $I=0, \ldots, M$, is performed once only, without any initializing or updating of the values of $\mathbf{d}_{i}$. The resulting algorithm is given below:

\section{Local Tikhonov Regularization Algorithm \#2}

1. Let $I=0$.

2. Holding the previously determined values of $c_{00}$, $c_{10}, \ldots, c_{I-1,0}$ fixed, solve for $\bar{\beta}$ :

$$
\min _{\beta}\left\|K_{R} \beta+\sum_{i=0}^{I-1} c_{i 0} \hat{\Delta}_{I-i+1}-\mathbf{f}_{I}\right\|_{R+1}^{2}+\alpha\|\beta\|_{R+1}^{2}
$$


3. Set $c_{I 0}=\bar{\beta}_{0}$, the first component of $\bar{\beta}$.

4. If $I=M$, stop. Otherwise, increment $I$ by 1 and return to step 2 .

In (Lamm and Eldén, 1995), this second algorithm is considered in detail, and convergence proofs for the numerical implementation are given in the case of the kernel satisfying $k(0)=0$. The more general case will be considered elsewhere. One can view this algorithm as a sequential Tikhonov regularization algorithm as follows: For $R \leq M$ (and, in general, we take $R$ much smaller than $M$ ), the matrix $K_{R}$ is a principal submatrix of the matrix $\mathbf{K}$

$$
\mathbf{K}=\left(\begin{array}{cccc}
\Delta_{1} & 0 & \ldots & 0 \\
\Delta_{2} & \Delta_{1} & \ldots & 0 \\
\vdots & \vdots & \ddots & \vdots \\
\Delta_{M+1} & \Delta_{M} & \ldots & \Delta_{1}
\end{array}\right)
$$

where $\mathbf{K}$ is the governing matrix in a standard collocation approximation, here collocating to $t_{1}, \ldots, t_{M+1}$ of the integral equation (1); that is, the collocation method applied to (1) leads to an equation of the form

$$
\mathbf{K q}=\mathbf{f}
$$

where $\mathbf{f} \equiv\left(f\left(t_{1}\right), \ldots, f\left(t_{M+1}\right)\right)^{\top}$ and where $q$ is the $(M+1)$-dimensional vector of coefficients in the finite dimensional approximation $u_{M}$ of $u$, given by

$$
u_{M}(t)=\sum_{i=0}^{M} q_{i} \chi_{i}(t), \quad t \in[0,1] .
$$

Standard (0th order) Tikhonov regularization for such a discretization consists of the minimization of $\|\mathbf{K q}-\mathbf{f}\|_{M+1}^{2}+\alpha\|\mathbf{q}\|_{M+1}^{2}$. With local (sequential) Tikhonov regularization, as given in the second algorithm above or in (Lamm and Eldén, 1995), one initially minimizes only the first $R+1$ components this problem, i.e., one solves for $\bar{\beta}$

$$
\min _{\beta}\left\|K_{R} \beta-\mathbf{f}_{0}\right\|_{R+1}^{2}+\alpha\|\beta\|_{R+1}^{2},
$$

and then sets $q_{0}=\bar{\beta}_{0}$, discarding the remaining components of $\bar{\beta}$. At the second step, the next $R+1$ components of the original problem are considered, and the following problem is solved for $\bar{\beta}$ :

$$
\min _{\beta}\left\|q_{0} \hat{\Delta}_{2}+K_{R} \beta-\mathbf{f}_{1}\right\|_{R+1}^{2}+\alpha\|\beta\|_{R+1}^{2},
$$

with then $q_{1}$ set equal to $\bar{\beta}_{0}$, and the rest of $\beta$ discarded. And so on.
Because $R$ is typically much smaller than $M$, and because the governing matrix $K_{R}$ is the same at every step, the computational cost is quite low, with the effectiveness about the same as full Tikhonov regularization. The reader is referred to (Lamm and Eldén, 1995) for numerical examples and for comparisons in terms of operation counts for standard Tikhonov regularization contrasted with an efficient implementation of the second algorithm above. The results in (Lamm and Eldén, 1995) are valid for collocation-type approximations as well as several standard methods based on the numerical quadrature of (1).

\section{CONVERGENCE ANALYSIS FOR PROBLEM $\mathcal{P}_{\Delta r}$}

We present here the convergence theory for the solution $\left(\eta_{\alpha, \Delta r}^{\delta}, \varphi_{\alpha, \Delta r}^{\delta}\right)$ of $\mathcal{P}_{\Delta r}$ as $\delta, \alpha, \Delta r$ go to zero in a prescribed way. First we will need the definition

$$
\omega(\Delta r)=\int_{0}^{\Delta r} \int_{0}^{\rho}|k(\rho-s)|^{2} d s d \rho,
$$

where we note that $\omega(\Delta r)=\mathcal{O}\left(\Delta r^{2}\right)$ as $\Delta r \rightarrow 0$, given our assumptions on $k$. For many ill-posed Volterra problems, $k$ is quite small near 0 , leading to the result that $\omega(\Delta r)$ converges to zero much more rapidly than $\mathcal{O}\left(\Delta r^{2}\right)$ in these cases.

Theorem 1 For each fixed $\Delta r \in(0, \Delta R]$ and fixed $\alpha>0$, there exists a unique solution $\left(\eta_{\alpha, \Delta r}^{\delta}, \varphi_{\alpha, \Delta r}^{\delta}\right)$ of problem $\mathcal{P}_{\Delta_{r}}$, where $F_{\Delta r}^{\delta} \in Z_{\Delta r}$ in problem $\mathcal{P}_{\Delta r}$ is defined by $F_{\Delta r}^{\delta}(t)(\rho)=f(t+r)+d(t+\rho), 0 \leq t \leq 1$, $0 \leq \rho \leq \Delta r$, with $\int_{0}^{1+\Delta r}|d(t)|^{2} d t \leq \delta^{2}$. Furthermore a selection of $\alpha=\alpha(\Delta r)>0$ may be made so that

(i) $\alpha(\Delta r) \rightarrow 0$, and

(ii) $\frac{\omega(\Delta r)}{\alpha(\Delta r)} \rightarrow 0$

as $\Delta r \rightarrow 0$, and a selection of $\Delta r=\Delta r(\delta)>0$ may be made such that

(iii) $\Delta r(\delta) \rightarrow 0$, and

(iv) $\frac{\delta^{2}}{\alpha(\Delta r(\delta))} \rightarrow 0$,

as $\delta \rightarrow 0$. For any sequence $\delta_{n} \rightarrow 0$, and for $\Delta r_{n} \equiv$ $\Delta r_{n}\left(\delta_{n}\right)$ and $\alpha_{n} \equiv \alpha\left(\Delta r\left(\delta_{n}\right)\right)$ such that (i)-(iv) hold, the corresponding solution $\left(\eta_{\alpha_{n}, \Delta r_{n}}^{\delta_{n}}, \varphi_{\alpha_{n}, \Delta r_{n}}^{\delta_{n}}\right)$ of $\mathcal{P}_{\Delta r_{n}}$ (defined using $F_{\Delta r_{n}}^{\delta_{n}}$ ) satisfies

$$
\eta_{\alpha_{n}, \Delta r_{n}}^{\delta_{n}} \rightarrow u \quad \text { as } n \rightarrow \infty .
$$


Proof: Define $\tilde{\mathcal{L}}_{\Delta r}(\eta, \varphi)=\mathcal{L}_{\Delta r} \varphi$ for all $(\eta, \varphi) \in H_{\Delta r}$ such that $\varphi \in \operatorname{dom}\left(\mathcal{L}_{\Delta r}\right)$. Restricting $\mathcal{C}_{\Delta r}$ and $\tilde{\mathcal{L}}_{\Delta r}$ to the Hilbert space $D_{\Delta r}$, we have the assumptions of (Locker and Prenter, 1980) holding for the problem $\mathcal{P}_{\Delta r}$ with $\mathcal{L}_{\Delta r} \varphi$ replaced by $\tilde{\mathcal{L}}_{\Delta r}(\eta, \varphi)$ (this problem is equivalent to $\mathcal{P}_{\Delta r}$ ); in particular, one has in this case that the nullspace of $\tilde{\mathcal{L}}_{\Delta r}$ is the trivial subspace and that the range of $\tilde{\mathcal{L}}_{\Delta r}$ is closed in $Z_{\Delta r}$. Thus, for each $\Delta r \in(0, \Delta R], \alpha>0$, and $F_{\Delta r}^{\delta} \in Z_{\Delta r}$, there exists a unique solution $\left(\eta_{\alpha, \Delta r}^{\delta}, \varphi_{\alpha, \Delta r}^{\delta}\right)$ of problem $\mathcal{P}_{\Delta r}$ given by the solution of the normal equations associated with problem $\mathcal{P}_{\Delta r}$.

We shall first show that, in the case of ideal data in problem $\mathcal{P}_{\Delta r}$ (i.e., $F_{\Delta r}$ appearing in place of $F_{\Delta r}^{\delta}$ ), the corresponding solutions $\left(\eta_{\alpha, \Delta r}, \varphi_{\alpha, \Delta r}\right)$ of problem $\mathcal{P}_{\Delta r}$ are bounded as $\Delta r \rightarrow 0$, provided $\alpha$ is chosen appropriately. From the definition of $\left(\eta_{\alpha, \Delta r}, \varphi_{\alpha, \Delta r}\right)$, one obtains $\eta_{\alpha, \Delta r}=T_{\Delta r} \varphi_{\alpha, \Delta r}$, where

$$
\begin{aligned}
& \alpha\left\|\varphi_{\alpha, \Delta r}\right\|_{\Delta r}^{2} \\
\leq & \left\|\mathcal{C}_{\Delta r}\left(\eta_{\alpha, \Delta r}, \varphi_{\alpha, \Delta r}\right)-F_{\Delta r}\right\|_{\Delta r}^{2}+\alpha\left\|\mathcal{L}_{\Delta r} \varphi_{\alpha, \Delta r}\right\|_{\Delta r}^{2} \\
\leq & \left\|\mathcal{C}_{\Delta r}\left(u, \hat{u}_{\Delta r}\right)-F_{\Delta r}\right\|_{\Delta r}^{2}+\alpha\left\|\mathcal{L}_{\Delta r} \hat{u}_{\Delta r}\right\|_{\Delta r}^{2} .
\end{aligned}
$$

Here $\hat{u}_{\Delta r} \in Z_{\Delta r}$ is defined by $\hat{u}_{\Delta r}(t)(\rho)=u(t), 0 \leq$ $t \leq 1,0 \leq \rho \leq \Delta r$, and thus $\left(u, \hat{u}_{\Delta r}\right) \in D_{\Delta r}$ (i.e., $\left.\ell\left(\hat{u}_{\Delta r}(t)\right)=u(t)\right)$. But

$$
\begin{aligned}
& \left\|\mathcal{C}_{\Delta r}\left(u, \hat{u}_{\Delta r}\right)-F_{\Delta r}\right\|_{\Delta r}^{2} \\
& =\frac{1}{\Delta r} \int_{0}^{1} \int_{0}^{\Delta r}\left[u(t) \int_{0}^{\rho} k(\rho-s) d s\right. \\
& \left.\quad+\int_{0}^{t} k(t+\rho-s) u(s) d s-f(t+\rho)\right]^{2} d \rho d t \\
& =\frac{1}{\Delta r} \int_{0}^{1} \int_{0}^{\Delta r}\left[\int_{0}^{\rho} k(\rho-s)(u(t)-u(t+s)) d s\right]^{2} d \rho d t \\
& \leq 4 \omega(\Delta r)\|u\|_{L_{2}(0,1+\Delta R)}^{2}
\end{aligned}
$$

where $\omega(\Delta r)$ is defined in (8). Thus

$$
\alpha\left\|\varphi_{\alpha, \Delta r}\right\|_{\Delta r}^{2} \leq 4 \omega(\Delta r)\|u\|_{L_{2}(0,1+\Delta R)}^{2}+\alpha\|u\|^{2}
$$

where we have used the fact that $\left\|\mathcal{L}_{\Delta r} \hat{u}_{\Delta r}\right\|_{\Delta r}^{2}=\|u\|^{2}$ $\left(\|\cdot\|\right.$ denotes the $L_{2}(0,1)$ norm). In addition, defining $\tilde{\varphi}_{\alpha, \Delta r} \in\left(Z,\|\cdot\|_{z}\right)$ via

$$
\tilde{\varphi}_{\alpha, \Delta r}(t)(\rho)=\varphi_{\alpha, \Delta r}(t)(\rho \Delta r), \quad 0 \leq t, \rho \leq 1,
$$

we have $\left\|\tilde{\varphi}_{\alpha, \Delta r}\right\|_{Z}^{2}=\left\|\varphi_{\alpha, \Delta r}\right\|_{\Delta r}^{2}$ for all $\Delta r \in(0, \Delta R]$, and, provided we select $\alpha=\alpha(\Delta r)$ such that $\frac{\omega(\Delta r)}{\alpha(\Delta r)}$ is bounded as $\Delta r \rightarrow 0$, we have that $\left\{\tilde{\varphi}_{\alpha, \Delta r}\right\} \subset Z$ is bounded for all $\Delta r \in(0, \Delta R]$, and therefore has a weakly converging subsequence.

Let $\left\{\Delta r_{n}\right\}$ denote a sequence which converges to zero as $n \rightarrow \infty$, and (relabeling the subsequence) let $\tilde{\varphi}_{n} \equiv$ $\tilde{\varphi}_{\alpha\left(\Delta r_{n}\right), \Delta r_{n}}$, where $\tilde{\varphi}_{n} \rightarrow \tilde{\varphi}_{0} \in Z$ as $n \rightarrow \infty$. (In what follows we will simplify notation wherever possible, and write $\mathcal{A}_{n} \equiv \mathcal{A}_{\Delta r_{n}}, \mathcal{B}_{n} \equiv \mathcal{B}_{\Delta r_{n}}, Z_{n} \equiv Z_{\Delta r_{n}},\|\cdot\|_{n} \equiv$ $\|\cdot\|_{\Delta r_{n}}, \varphi_{n} \equiv \varphi_{\alpha\left(\Delta r_{n}\right), \Delta r_{n}}, \alpha_{n} \equiv \alpha\left(\Delta r_{n}\right)$, etc.) Defining $\eta_{0} \in L_{2}(0,1)$ via $\eta_{0}=T \tilde{\varphi}_{0}$, we note that $\eta_{n} \equiv T_{n} \varphi_{n}=$ $T \tilde{\varphi}_{n}$ satisfies $\eta_{n} \rightarrow \eta_{0}$ as $n \rightarrow 0$.

First we show that $\tilde{\varphi}_{0}$ is a least squares solution of a related problem,

$$
\tilde{\mathcal{A}} \tilde{\varphi}=f
$$

where $\tilde{\mathcal{A}} \in \mathcal{L}\left(Z, L_{2}(0,1)\right)$ is given by

$$
\tilde{\mathcal{A}} \tilde{\varphi}(t)=\int_{0}^{t} k(t-s)(T \tilde{\varphi})(s) d s, t \in[0,1]
$$

for $\tilde{\varphi} \in Z$. To this end we note that

$$
\begin{aligned}
& \left\|\tilde{\mathcal{A}} \tilde{\varphi}_{0}-f\right\|^{2} \\
= & \int_{0}^{1}\left|\int_{0}^{t} k(t-s) \eta_{0}(s) d s-f(t)\right|^{2} d t \\
= & \frac{1}{\Delta r_{n}} \int_{0}^{1} \int_{0}^{\Delta r_{n}}\left|A \eta_{0}(t)-f(t)\right|^{2} d \rho d t \\
= & \left\|\hat{A}_{n} \eta_{0}-\hat{F}_{n}\right\|_{n}^{2}
\end{aligned}
$$

where $\hat{A}_{n}: L_{2}(0,1) \rightarrow Z_{n}$ is given for $\eta \in L_{2}(0,1)$ by $\hat{A}_{n} \eta(t)(\rho)=A \eta(t), t \in[0,1], \rho \in\left[0, \Delta r_{n}\right]$, and $\hat{F}_{n} \in Z_{n}$ is given by $\hat{F}_{n}(t)(\rho)=f(t)$. We thus have, for some $C>0$ independent of $n$ and $\Delta r_{n}$,

$$
\begin{aligned}
&\left\|\tilde{\mathcal{A}} \tilde{\varphi}_{0}-f\right\|^{2} \\
& \leq \quad C\left\{\left\|\mathcal{A}_{n} \varphi_{n}+\mathcal{B}_{n} \eta_{n}-F_{n}\right\|_{n}^{2}+\left\|\mathcal{A}_{n} \varphi_{n}\right\|_{n}^{2}\right. \\
&+\left\|\mathcal{B}_{n} \eta_{n}-\hat{A}_{n} \eta_{0}\right\|_{n}^{2}+\left\|F_{n}-\hat{F}_{n}\right\|_{n}^{2} \\
&\left.+\alpha_{n}\left\|\varphi_{n}\right\|_{n}^{2}\right\} .
\end{aligned}
$$

But $\left(\eta_{n}, \varphi_{n}\right)$ a solution of $\mathcal{P}_{\Delta r_{n}}$ implies that

$$
\begin{aligned}
& \left\|\mathcal{A}_{n} \varphi_{n}+\mathcal{B}_{n} \eta_{n}-F_{n}\right\|_{n}^{2}+\alpha_{n}\left\|\varphi_{n}\right\|_{n}^{2} \\
& \quad \leq\left\|\mathcal{A}_{n} \hat{u}_{n}+\mathcal{B}_{n} u-F_{n}\right\|_{n}^{2}+\alpha_{n}\left\|\hat{u}_{n}\right\|_{n}^{2} \\
& \quad \leq 4 \omega\left(\Delta r_{n}\right)\|u\|_{L_{2}(0,1+\Delta R)}^{2}+\alpha_{n}\|u\|^{2}
\end{aligned}
$$

using earlier arguments and $\hat{u}_{n} \equiv \hat{u}_{\Delta r_{n}}$, where $\hat{u}$ was defined after equation (9). In addition,

$$
\begin{aligned}
& \left\|\mathcal{B}_{n} \eta_{n}-\hat{A}_{n} \eta_{0}\right\|_{n}^{2} \\
& \quad \leq 2\left\|\left(\mathcal{B}_{n}-\hat{A}_{n}\right) \eta_{n}\right\|_{n}^{2}+2\left\|\hat{A}_{n}\left(\eta_{n}-\eta_{0}\right)\right\|_{n}^{2} \\
& \quad \leq \frac{2}{\Delta r_{n}}\left\|\eta_{n}\right\|^{2} \int_{0}^{1} \int_{0}^{\Delta r_{n}} \int_{0}^{t}|k(t+\rho-s)-k(t-s)|^{2} d s d \rho d t
\end{aligned}
$$




$$
\begin{aligned}
& +\frac{2}{\Delta r_{n}} \int_{0}^{1} \int_{0}^{\Delta r_{n}}\left|A\left(\eta_{n}-\eta_{0}\right)(t)\right|^{2} d \rho d t \\
\leq & \frac{2}{3} L_{k}^{2}\|T\|^{2}\left\|\tilde{\varphi}_{n}\right\|_{Z}^{2} \Delta r_{n}^{2} \\
& +2\left\|A\left(\eta_{n}-\eta_{0}\right)\right\|^{2}
\end{aligned}
$$

so that, using the compactness of the operator $A$ in the second term, $\left\|\mathcal{B}_{n} \eta_{n}-\hat{A}_{n} \eta_{0}\right\|_{n}^{2} \rightarrow 0$ as $n \rightarrow \infty$. In addition,

$$
\begin{aligned}
& \left\|F_{n}-\hat{F}_{n}\right\|_{n}^{2} \\
= & \frac{1}{\Delta r_{n}} \int_{0}^{1} \int_{0}^{\Delta r_{n}}|f(t+\rho)-f(t)|^{2} d \rho d t \\
\leq & \frac{1}{3} L_{f}^{2} \Delta r_{n}^{2}
\end{aligned}
$$

while boundedness of $\left\|\varphi_{n}\right\|_{n}^{2}$ as $n \rightarrow \infty$ gives $\left\|\mathcal{A}_{n} \varphi_{n}\right\|_{n}^{2}=\mathcal{O}\left(\Delta r_{n}^{2}\right)$. Thus, letting $n \rightarrow 0$ in (10) and assuming $\alpha=\alpha(\Delta r)$ satisfies (i) and (ii) in the statement of the theorem (this is always possible), we find that

$$
\left\|\tilde{\mathcal{A}} \tilde{\varphi}_{0}-f\right\|^{2}=0,
$$

so that $\tilde{\varphi}_{0} \in Z$ is a least squares solution of $\mathcal{A} \varphi=f$.

Now let $\tilde{u} \in Z$ be given by

$$
\tilde{u} \equiv \frac{u(t)}{\ell(\gamma)} \gamma, \quad t \in[0,1],
$$

where $\gamma$ was defined earlier. Then

$$
\begin{aligned}
\tilde{\mathcal{A}} \tilde{u}(t) & =\int_{0}^{t} k(t-s) \ell\left(\frac{u(s)}{\ell(\gamma)} \gamma\right) d s \\
& =f(t), \quad \text { a.a. } t \in[0,1]
\end{aligned}
$$

and one can show that $\tilde{u}$ is in $\mathcal{N}(\tilde{\mathcal{A}})^{\perp}($ where $\mathcal{N}(\tilde{\mathcal{A}})$ denotes the nullspace of $\tilde{\mathcal{A}})$. Such conditions give that $\tilde{u}$ is the (unique) minimum-norm least squares solution of $\tilde{\mathcal{A}} \tilde{\varphi}=f$, (see, e.g., (Groetsch, 1984)). Our claim here is that the weak-limit $\tilde{\varphi}_{0}$ of $\tilde{\varphi}_{n}$ is actually equal to $\tilde{u}$; to show this we need only argue that $\left\|\tilde{\varphi}_{0}\right\|_{z}=\|\tilde{u}\|_{z}$. We have

$$
\begin{aligned}
\alpha_{n}\left\|\tilde{\varphi}_{n}\right\|_{Z}^{2} & =\alpha_{n}\left\|\varphi_{n}\right\|_{n}^{2} \\
& \leq\left\|\mathcal{A}_{n} \varphi_{n}+\mathcal{B}_{n} \eta_{n}-F_{n}\right\|_{n}^{2}+\alpha_{n}\left\|\varphi_{n}\right\|_{n}^{2} \\
& \leq\left\|\mathcal{A}_{n} \tilde{u}_{n}+\mathcal{B}_{n} u-F_{n}\right\|_{n}^{2}+\alpha_{n}\left\|\tilde{u}_{n}\right\|_{n}^{2}
\end{aligned}
$$

where $\left(u, \tilde{u}_{n}\right) \in D_{n}$, and where $\tilde{u}_{n} \in Z_{n}$ is defined from $\tilde{u}$ in $(11)$ via $\tilde{u}_{n}(t)(\rho)=\tilde{u}(t)\left(\rho / \Delta r_{n}\right), 0 \leq t \leq 1$, $0 \leq \rho \leq \Delta r_{n}$. But

$$
\begin{aligned}
\left\|\mathcal{A}_{n} \tilde{u}_{n}+\mathcal{B}_{n} u-F_{n}\right\|_{n}^{2} & =\left\|\mathcal{A}_{n}\left(\tilde{u}_{n}-u_{n}\right)\right\|_{n}^{2} \\
& \leq 2 C \omega\left(\Delta r_{n}\right)\|u\|_{L_{2}(0,1+\Delta R)}^{2}
\end{aligned}
$$

where we have used $u_{n}=u_{\Delta r_{n}}\left(u_{\Delta r}\right.$ defined in (2)) and where $C=C\left(\|\gamma\|_{\infty}\right)$. In addition, $\left\|\tilde{u}_{n}\right\|_{n}^{2}=\|\tilde{u}\|_{z}^{2}=$ $\|u\|^{2} /\|\gamma\|^{2}$, and thus

$$
\left\|\tilde{\varphi}_{n}\right\|_{Z}^{2} \leq 2 C \frac{\omega\left(\Delta r_{n}\right)}{\alpha\left(\Delta r_{n}\right)}\|u\|_{L_{2}(0,1+\Delta R)}^{2}+\|u\|^{2} /\|\gamma\|^{2} .
$$

Selecting $\alpha(\Delta r)$ such that $\omega(\Delta r) / \alpha(\Delta r) \rightarrow 0$ as $\Delta r \rightarrow$ 0 , and letting $n \rightarrow \infty$ in the above estimate, we have

$$
\begin{aligned}
\left\|\tilde{\varphi}_{0}\right\|_{z} \leq & \liminf \left\|\tilde{\varphi}_{n}\right\|_{z} \\
\leq & \lim \sup \left\|\tilde{\varphi}_{n}\right\|_{z} \\
\leq & \|u\| /\|\gamma\| \\
& =\|\tilde{u}\|_{z} .
\end{aligned}
$$

By uniqueness of the minimum norm least squares solution, it must be that $\tilde{\varphi}_{0}=\tilde{u}$ and, from (12), that $\left\|\tilde{\varphi}_{n}\right\|_{z} \rightarrow\|\tilde{u}\|_{z}$ as $n \rightarrow \infty$. Thus we have strong convergence of $\tilde{\varphi}_{n}$ to $\tilde{u}$ as $n \rightarrow \infty$, in fact, uniqueness of the limit $\tilde{u}$ gives full sequential convergence for arbitrary $\left\{\Delta r_{n}\right\}$ such that $\Delta r_{n} \rightarrow 0$ as $n \rightarrow \infty$.

Finally, $T \tilde{\varphi}_{n} \rightarrow T \tilde{u}$ in $L_{2}(0,1)$ as $n \rightarrow \infty$, or, equivalently, $\eta_{n} \rightarrow \eta_{0}=T \tilde{u}=u$.

Now let $\left(\eta_{\alpha, \Delta r}^{\delta}, \varphi_{\alpha, \Delta r}^{\delta}\right)$ and $\left(\eta_{\alpha, \Delta r}, \varphi_{\alpha, \Delta r}\right)$, denote the solutions of $\mathcal{P}_{\Delta r}$ associated with data $F_{\Delta r}^{\delta}$ and $F_{\Delta r}$, respectively, for given $\Delta r \in(0, \Delta R]$. Completely standard arguments (see, for example, Section 1.4 of (Morozov, 1984)) may be used to show that

$$
\begin{gathered}
\frac{1}{2}\left\|\mathcal{C}_{\Delta r}\left(\eta_{\alpha, \Delta r}^{\delta}-\eta_{\alpha, \Delta r}, \varphi_{\alpha, \Delta r}^{\delta}-\varphi_{\alpha, \Delta r}\right)\right\|_{\Delta r}^{2} \\
\quad+\alpha\left\|\varphi_{\alpha, \Delta r}^{\delta}-\varphi_{\alpha, \Delta r}\right\|_{\Delta r}^{2} \leq \frac{1}{2}\left\|d_{\Delta r}\right\|_{\Delta r}^{2}
\end{gathered}
$$

where $d_{\Delta r}(t)(\rho)=d(t+\rho)$ for $t \in[0,1], \rho \in[0, \Delta r]$, that is,

$$
\left\|\varphi_{\alpha, \Delta r}^{\delta}-\varphi_{\alpha, \Delta r}\right\|_{\Delta r}^{2} \leq \frac{\delta^{2}}{2 \alpha} .
$$

The remainder of the theorem then follows from an application of the triangle inequality and from the definition of $\eta_{\alpha, \Delta r}^{\delta}=T_{\Delta r} \varphi_{\alpha, \Delta r}^{\delta}$.

\section{References}

[1] Auslender, A., 1971, "Methódes numériques pour la décomposition et la minimisation de fonctions non différentiables," Numerische Mathematik, Vol. 18, pp. 213-223. 
[2] Gripenberg, G., Londen, S.-O., and Staffans, O., 1990, Volterra Integral and Functional Equations, Cambridge University Press, Cambridge.

[3] Groetsch, C. W., 1984, The Theory of Tikhonov Regularization for Fredholm Equations of the First Kind, Pitman, London.

[4] Lamm, P. K., 1995, "Future-Sequential Regularization Methods for Ill-Posed Volterra Equations," Journal of Mathematical Analysis and Applications, to appear.

[5] Lamm, P. K., and Eldén, L., 1995, "Numerical Solution of First-Kind Volterra Equations by Sequential Tikhonov Regularization," preprint.

[6] Locker, J., and Prenter, P. M., 1980, "Regularization with Differential Operators, I. General Theory," Journal of Mathematical Analysis and Applications, Vol. 74, pp. 504-529.

[7] Morozov, V. A., 1984, Methods for Solving Incorrectly Posed Problems, Springer, Berlin. 